\title{
Determining mild, moderate, and severe pain equivalency across pain- intensity tools in nursing home residents
}

\author{
Katherine R. Jones, RN, PhD, FAAN; ${ }^{1}$ Carol P. Vojir, PhD; ${ }^{1}$ Evelyn Hutt, MD; ${ }^{2 *}$ Regina Fink, RN, PhD, FAAN ${ }^{3}$ \\ ${ }^{1}$ School of Nursing, University of Colorado at Denver and the Health Sciences Center, Denver, CO; ${ }^{2}$ Denver Depart- \\ ment of Veterans Affairs Medical Center, Research Enhancement Award Program; School of Medicine, University of \\ Colorado at Denver and the Health Sciences Center, Denver, CO; ${ }^{3}$ University of Colorado Hospital, Denver, CO
}

\begin{abstract}
Older adults in nursing homes experience pain that is often underassessed and undertreated. Visual analog painintensity scales, recommended for widespread use in adults, do not work well in the older adult population. A variety of other tools are in use, including the Verbal Descriptor Scale, the Faces Pain Scale (FPS), and the Numeric Rating Scale. These tools are more acceptable to older adults, but no agreement exists about how to compare the resulting pain-intensity scores across residents. This study examined the equivalency of painintensity scores for 135 nursing home residents who reported their pain on the three different instruments. The results were validated with a second sample of 135 nursing home residents. The pain levels across the three tools were highly correlated, but residents were found to underrate higher pain intensity on the FPS. A modification of scoring for the FPS led to greater agreement across the three tools. The findings have implications for use of these tools for quality improvement and public reporting of pain.
\end{abstract}

Key words: dementia, elderly, Faces Pain Scale, Numeric Rating Scale, nursing homes, pain assessment, pain intensity, pain-intensity tools, pain-level equivalency, rehabilitation.

\section{INTRODUCTION}

Pain is common among older adults because of the increase in chronic health problems that occurs with aging [1-3]. Nursing home residents in particular experience more pain than older adults who are not in nursing home settings [1]. As many as 66 percent of nursing home residents experience constant pain [4]; at least 40 percent have daily pain, 71 percent any type of pain [5], 83 percent chronic pain [6], and 28 percent excruciating pain within the previous 7 days [7]. Undertreated pain has been estimated to affect 45 to 80 percent of nursing home residents [8].

Accurate pain assessment is a prerequisite for successful pain management [9]. The American Pain Society (APS) and American Geriatric Society (AGS) both emphasize the importance of obtaining the patient's selfreport of pain, the gold standard of pain assessment $[8,10$ 11]. Although pain is a multidimensional concept, subjective intensity is probably the component most often measured in clinical practice and pain management outcomes research [12-14]. Multiple tools exist for the measurement or quantification of pain severity or intensity: visual analog scales (VASs), the Verbal Descriptor Scale (VDS), the Numeric Rating Scale (NRS) (0-10 points, horizontal or vertical), the Faces Pain Scale (FPS) (six or seven

\footnotetext{
Abbreviations: AGS = American Geriatric Society, APS = American Pain Society, FPS = Faces Pain Scale, NRS = Numeric Rating Scale, PT = Pain Thermometer, RA = research assistant, VAS = visual analog scale, VDS = Verbal Descriptor Scale.

*Address all correspondence to Evelyn Hutt, MD; Director of Research in Care Coordination, Denver VAMC, 151, 1055 Clermont Street, Denver, CO 80220; 303-370-7576; fax: 303-370-7577. Email: evelyn.hutt@uchsc.edu DOI: 10.1682/JRRD.2006.05.0051
} 
faces, with or without tears), the Color Pain Assessment scale, and the Pain Thermometer (PT). The 0-10 NRS scale has been recommended for universal adoption in clinical assessment of pain intensity in adults [15], but it does not work particularly well for older adults. Studies suggest that individuals with cognitive impairment, who represent the overwhelming majority of nursing home residents, find self-report instruments that have written word cues the easiest and VASs the most difficult to use [9]. Several studies have demonstrated that older adults prefer the VDS when given a choice $[9,14,16]$. However, Jones et al. also reported differences among subgroups of older adults in their preferences for pain scales: males preferred the NRS significantly more often than females, while older Hispanic adults preferred the FPS significantly more often than nonminority groups [16]. Patientcentered care suggests that individuals should be given a choice of pain-intensity tools when reporting their pain, but no method for establishing pain-intensity equivalency across these tools exists.

The AGS recommends that "a quantitative assessment of pain should be recorded by the use of a standard pain scale" $[8,17]$ and specifically mentions the VDS, PT, NRS, and FPS. The American Medical Directors Association clinical practice guideline also recommends using a standardized scale to quantify the intensity of the patient's pain at its best and worst and includes a table showing the VDS, NRS, and a VAS [18]. These scales, however, contain varying numbers of pain levels and different wording for the pain-intensity levels and result in varying ranges of pain-intensity scores, all of which make comparing painintensity levels across instruments difficult. If multiple tools are used for measuring pain intensity within or across settings, we need to know how these various rating systems can be reliably and validly converted to a single simplified metric, such as none, mild, moderate, or severe pain. Also, determining whether the scaling system from the nationally standardized nursing home resident assessment tool, the Minimum Data Set (mild, moderate, or excruciating pain), is equivalent to the more traditional pain metric used by clinicians is important.

So far, emphasis in the pain literature has been on establishing valid cutoff points for mild, moderate, and severe pain on the NRS only. The literature includes studies that have attempted to identify pain-intensity cutoff points by pain type and diagnosis using cancer-related pain [19-20], chronic pain [21-23], and acute pain conditions [24]. Serlin et al. developed a method to identify numeric cutoff points for levels of metastatic cancerrelated pain in multiple cultures by using patient selfreport of worst pain and its interference with various activities (enjoyment of life, activity, walking, mood, sleep, work, and relations with others) on a $0-10(0=$ does not interfere and $10=$ interferes completely) scale [20]. As shown in Table 1, responses clustered into three ranges: 1-4 for mild pain, 5-6 for moderate pain, and 7-10 for severe pain. These authors noted that the relationship between pain and interference with function was not linear. The steps between 4 and 5 and between 6 and 7 in pain severity on the $0-10$ NRS are more significant than other steps in terms of pain interference with function; pain began to interfere with life at level 5 . The same cutoff points were reported by Mendoza et al. [24] and Turner et al. [22]. Other researchers, however, using different populations, types of pain, and pain-reference points (average rather than worst pain) have reported cutoff points for mild, moderate, and severe pain that vary from those of Serlin et al. [20]. As shown in Table 1, Palos and colleagues reported cutoff points using decimals [25]; Paul et al. found the same upper boundary for mild pain but a different upper boundary for moderate pain [19]; Zelman et al. also found the same cutoff point for mild pain but different cutoff points for moderate pain when comparing patients with low back pain versus those with osteoarthritis [23]. Jensen et al. found the same cutoff points as Serlin et al. for back pain but not for phantom limb pain or pain in general [21].

While these studies have helped advance our ability to standardize mild, moderate, and severe pain categories across selected types of pain and diagnoses, they do not help us understand how to translate verbal or graphic intensity scales into equivalent standardized nomenclature. The cutoff points may also not be relevant for use with older adults who are experiencing both acute and chronic pain from multiple comorbid conditions. Thus, this analysis builds on and extends the work already done on establishing cutoff points for mild, moderate, and severe pain for individual scales and the equivalency in these categorizations across multiple scales.

\section{COMPARISON ACROSS PAIN-INTENSITY SCALES}

Most of the studies comparing pain-intensity scales have focused on comprehension levels and completion 
Table 1.

Summary of previous research on pain-intensity categorizations and cutoff points between mild, moderate, and severe pain levels.

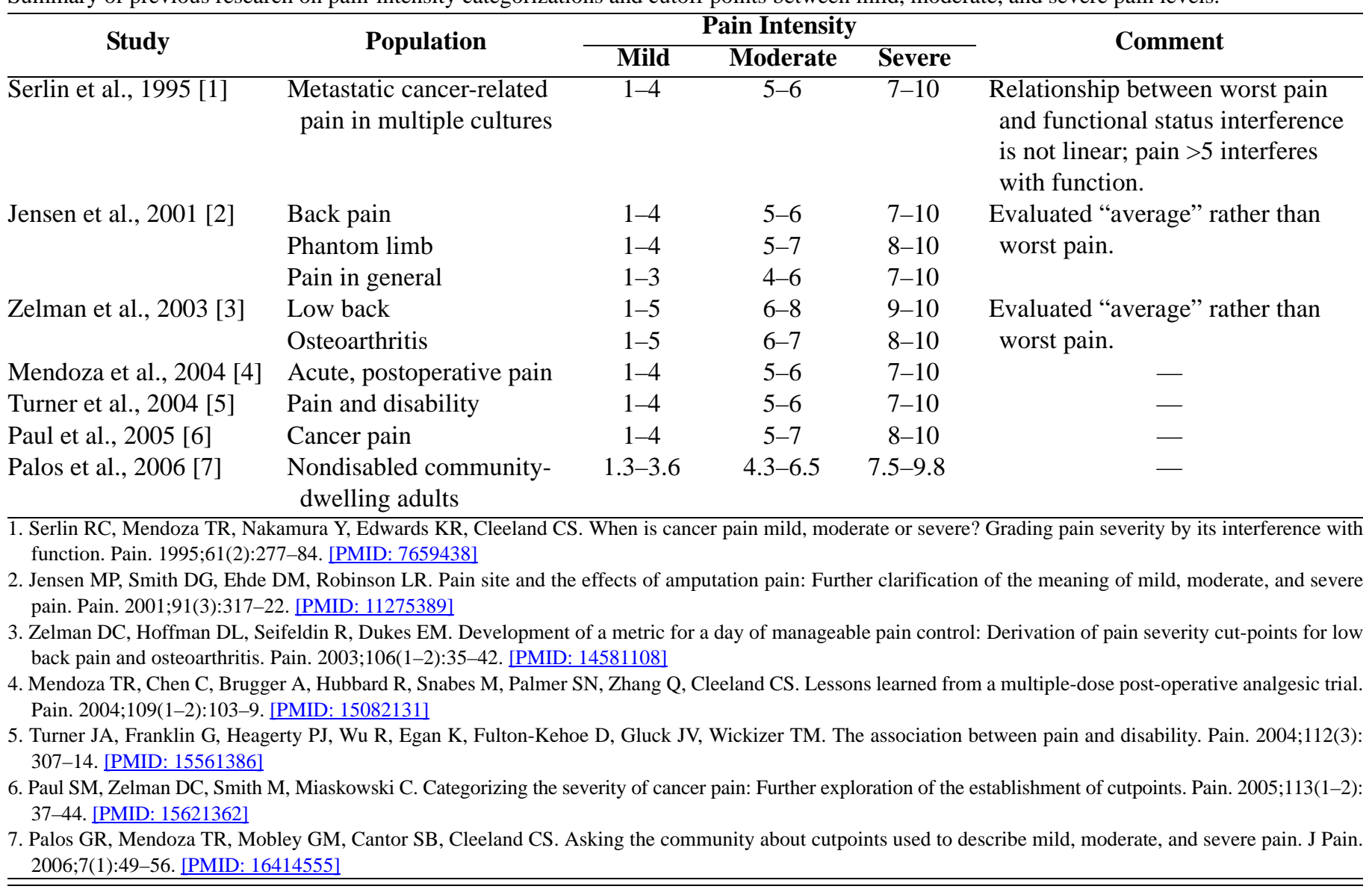

rates. Little work has been done thus far on translating the pain-intensity scores obtained by the different tools into a common metric. Instead, investigators have made assumptions about what the various intensity levels represent. For example, Krulewitch et al. compared subjects reporting moderate to severe pain with those reporting no or minimal pain using three different tools [26]. They defined moderate to severe pain as a score of $>1$ on the FPS (scores ranged from $0-6$ ) and $>25$ percent on a VAS line. No rationale was provided for these cutoff points.

Herr and Mobily compared five different painintensity tools in a sample of older adults [14]. The five scales correlated significantly, but the authors cautioned that they cannot be assumed to be equivalent. The horizontal VAS had lower pain-intensity scores compared with the vertical VAS. No significant differences between the vertical VAS and the other tools (VDS, NRS, and PT) were found. However, subjects were primarily in mild pain, well-educated, and white.
In a recent study, Jones et al. converted pain-intensity scores associated with the Bieri FPS [27], NRS, and VDS into four levels (none, mild, moderate, and severe) to analyze the effectiveness of a pain intervention [16]. Decisions regarding the assignment of pain-intensity level (which face, words, or number) to the 4-level metric were made after extensive review of the pain literature, consultation with several pain experts, research team discussion and consensus generation, and consideration of pain guideline recommendations. The assignments were as follows:

- NRS: $0=$ no pain, $1-3=$ mild pain, $4-6=$ moderate pain, and 7-10 = severe pain.

- VDS: no pain; slight and mild pain = mild pain; moderate pain; severe pain, very severe pain, and most intense pain possible $=$ severe pain.

- Bieri FPS: face 1 = no pain, faces $2-3$ = mild pain, faces $4-5=$ moderate pain, faces $6-7=$ severe pain. 
Jones et al. found that subjects who selected the NRS to report their pain intensity were significantly more likely to report moderate or severe pain compared with subjects who selected either of the other two pain-intensity tools [16]. They could not determine whether those with higher pain-intensity levels more often selected the NRS or whether use of the NRS led to systematically greater numbers of subjects being categorized at these higher pain levels. This study was conducted to explore this issue.

\section{METHODS}

The parent intervention study ( $n=2,034$ interviews of 1,182 residents over 27 months) was designed to improve pain practices in nursing homes. The overall methodology for the study is presented in detail elsewhere [28-29]. In short, research assistants (RAs) conducted a quarterly pain interview on a 20 percent random sample of nursing home residents living in 12 nursing homes in one state. Residents who were unable to be interviewed were included in the study and observed for signs of pain. For the first 6 assessments (18 months), residents were given a choice of which pain-intensity tool to use (NRS, Bieri FPS, or VDS). The Bieri version of the FPS was chosen [27], and permission for its use was obtained from the publisher. For the last three quarterly assessments (9 months), subjects were asked to report their pain using all three tools. In addition, they were asked to express a preference of tool. The purpose was to equate the three tools, knowing that individuals and clinicians prefer and use different tools for reporting painintensity levels.

The procedures for the last three quarterly pain assessments were as follows. After obtaining written informed consent from the residents or witnessed verbal consent from their legal guardians (as approved by the appropriate investigational review board), study RAs asked subjects whether or not they were experiencing pain now or had experienced pain in the past 24 hours. If the answer was yes (either immediately or after several probes using synonyms for the word "pain"), the RA asked the resident to specify the intensity of that pain, both current and the worst level in the past day, using three different pain intensity tools (VDS, NRS, and Bieri FPS). These scales were printed in large type, each on a separate piece of paper, and were presented in random order across subjects. After providing the intensity infor- mation, residents were asked to express a preference for a pain-intensity tool. The RA then completed the brief pain interview to the extent possible given the cognitive status of the resident, conducted a chart review for pain-related information, and observed the resident for specific pain behaviors when at rest and when active. All data were coded and entered into a Statistical Package for the Social Sciences (SPSS Inc, Chicago, Illinois) database.

A total of 647 resident interviews were completed during the final three quarters ( 9 months) of the study. Of the 510 residents sampled once, 270 (52.9\%) completed pain-intensity assessments using all three scales. The 270 residents who had been sampled once during this 9-month data collection period and whose responses were complete were randomly split into two samples, one for establishing pain-intensity equivalence (equivalence sample) and one for validating equivalence (validation sample). We used frequencies, cross-tabulations with chi-square tests, correlations, and simple linear regressions to establish and validate the equivalence between scales. No assumptions for the parametric regression technique were violated. The VDS was used as the standard against which equivalence for the NRS and Bieri FPS was determined because previous studies have shown it to be the preferred pain-intensity scale for use among older adults.

We then categorized each scale into the 4-level metric described previously to provide a standard metric using clinical relevance for comparison. Overall average percent agreement was then calculated by cross-tabulating the categorized NRS and Bieri FPS scales separately against the categorized VDS and computing the percentage of agreement in the no pain, mild pain, moderate pain, and severe pain categories (agreeing residents/total residents, expressed as a percent).

\section{RESULTS}

Agreement (consistency) among the scales was estimated on data collected from the 377 residents who had been resampled at least once during the three quarterly data collection periods. Only four residents provided results for all scales at all three quarterly data collection periods so test-retest analyses across scales and data collection points were not possible. Instead, we calculated two separate cross-tabulations of the categorized NRS and Bieri FPS scores against the VDS to assess overall average agreement among the scales for each of the three testing periods, for a 
total of six analyses. Averaging the overall agreement for a testing period produced three proxy measures of consistency, 70.4 percent $(n=22), 63.9$ percent $(n=18)$, and 82.2 percent $(n=14)$ for the three periods, respectively. All six chi-square statistics were significant at the 0.01 level.

As mentioned previously, a total of 647 resident interviews were completed during the final three quarters of the study. Of the 510 residents sampled only once during the final three quarters, 270 (52.9\%) completed pain-intensity assessments using all three scales. The rest were unable or unwilling to report their pain using all three tools. These 270 residents differed significantly from residents who were sampled more than once only in that they had shorter lengths of stay $(p=0.03)$ and thus were more likely not to have been available for earlier pain assessments. The 270 residents were randomly split into two samples of 135, one for equivalence testing (equivalence sample) and the second for validation of the results (validation sample). Table 2 shows the demographic characteristics for (1) parent study residents sampled once, (2) residents sampled once in the last three quarters of the study, and (3) residents in the equivalence and validation samples. The table also provides a comparison of the original scale mean and standard deviation values between the equivalence and validation samples. No significant differences were noted between equivalence and validation samples on any demographic or scale variable $(p<0.05)$. Intercorrelation among the three scales was $>0.8(p<0.001)$ for both samples.

Table 3 shows the correspondence between the categorized VDS and categorized NRS and Bieri FPS scores. These cross-tabulations showed highly significant association between tools $(p<0.001)$. Between the VDS and the NRS, overall average percent agreement, calculated as described previously, was 70.4 percent; between the VDS and the Bieri FPS, overall average percent agreement was 68.9 percent. Two aspects of Table 3 are notable: first, residents scored mild and moderate pain on the VDS in higher NRS categories, and second, residents scored mild pain on the VDS in higher Bieri FPS categories but moderate pain on the VDS in lower Bieri FPS categories. This tendency to underrate higher intensity pain on the FPS is also demonstrated in the markedly poor agreement between the severe pain categories of the two scales.

Simple linear regression analyses that predicted raw NRS and raw Bieri FPS scores from raw VDS scores yielded significant $r^{2}$ values of 0.795 and 0.747 ( $p<$ 0.001 ), respectively. Several additional variables (dementia diagnosis [yes or no], age over 85 [yes or no], and Hispanic status [yes or no]) were also evaluated for their contributions to the regression; none contributed significantly, thus no additional variables were used in the regressions. Using the regression equations for raw data and the VDS cutoff points previously described, we predicted the integer cutoff points for the NRS and Bieri FPS categories as follows:

- NRS: $0=$ no pain, 1-4 (note change) = mild pain, 5-6 (note change) $=$ moderate pain, $7-10=$ severe pain .

- Bieri FPS: face 1 = no pain, faces $2-3=$ mild pain, face 4 (note change) = moderate pain, faces $5-7$ (note change $)=$ severe pain .

The use of these predicted cutoff points to recategorize the raw data yielded the percent agreement shown in Table 4. Overall average percent agreement was 69.6 percent for the NRS, which indicates no improvement over

Table 2.

Sample demographics and scale statistics for parent study sample, analysis subsample (last three quarters), equivalence sample, and validation sample.

\begin{tabular}{|c|c|c|c|c|}
\hline Variable & $\begin{array}{l}\text { Parent Study Sample } \\
(n=1,182)\end{array}$ & $\begin{array}{l}\text { Last Three Quarters } \\
\qquad(n=510)\end{array}$ & $\begin{array}{l}\text { Equivalence Sample } \\
\qquad(n=135)\end{array}$ & $\begin{array}{c}\text { Validation Sample } \\
(n=135)\end{array}$ \\
\hline Female (\%) & 68.9 & 68.0 & 72.6 & 72.6 \\
\hline Caucasian (\%) & 84.8 & 87.8 & 87.4 & 87.4 \\
\hline Prefer VDS (\%) & 51.0 & 48.4 & 49.5 & 44.6 \\
\hline Dementia Dx (\%) & 42.9 & 35.3 & 26.7 & 25.6 \\
\hline LOS (d) (mean \pm SD) & $645.8 \pm 1157.2$ & $739.6 \pm 1322.9$ & $508.5 \pm 779.7$ & $613.8 \pm 1266.8$ \\
\hline VDS (mean \pm SD) & - & - & $1.9 \pm 1.6$ & $1.9 \pm 1.9$ \\
\hline NRS (mean \pm SD) & - & - & $3.5 \pm 2.9$ & $3.3 \pm 3.1$ \\
\hline FPS (mean \pm SD) & - & - & $1.9 \pm 1.5$ & $1.9 \pm 1.7$ \\
\hline
\end{tabular}


JRRD, Volume 44, Number 2, 2007

Table 3.

Agreement $^{*}$ between Verbal Descriptor Scale (VDS) and original Numeric Rating Scale (NRS) and Bieri Faces Pain Scale (FPS) (equivalence sample).

\begin{tabular}{|c|c|c|c|c|c|}
\hline \multirow{2}{*}{ VDS } & \multicolumn{5}{|c|}{ Original NRS } \\
\hline & No Pain & Mild Pain & Moderate Pain & Severe Pain & Total \\
\hline No Pain & $37(90.2 \%)$ & 4 & 0 & 0 & 41 \\
\hline Mild Pain & 1 & 22 (56.4\%) & 15 & 1 & 39 \\
\hline Moderate Pain & 0 & 3 & $22(68.8 \%)$ & 7 & 32 \\
\hline Severe Pain & 0 & 0 & 9 & $14(60.9 \%)$ & 23 \\
\hline Total & 38 & 29 & 46 & 22 & 135 \\
\hline No Pain & $40(97.6 \%)$ & 1 & 0 & 0 & 41 \\
\hline Mild Pain & 2 & $28(71.8 \%)$ & 9 & 0 & 39 \\
\hline Moderate Pain & 0 & 9 & $22(68.8 \%)$ & 1 & 32 \\
\hline Severe Pain & 0 & 2 & 18 & 3 (13.0\%) & 23 \\
\hline Total & 42 & 40 & 49 & 4 & 135 \\
\hline
\end{tabular}

Table 4.

Agreement $^{*}$ between Verbal Descriptor Scale (VDS) and recategorized Numeric Rating Scale (NRS) and Bieri Faces Pain Scale (FPS) from regression results (equivalence sample).

\begin{tabular}{lccccc}
\hline \multicolumn{1}{c}{ VDS } & \multicolumn{5}{c}{ Recategorized NRS } \\
\cline { 2 - 6 } & No Pain & Mild Pain & Moderate Pain & Severe Pain & Total \\
\hline No Pain & $37(90.2 \%)$ & 4 & 0 & 0 & 41 \\
Mild Pain & 1 & $27(69.2 \%)$ & 10 & 1 & 39 \\
Moderate Pain & 0 & 9 & $16(50.0 \%)$ & 7 & 32 \\
Severe Pain & 0 & 1 & 8 & $14(60.9 \%)$ & 23 \\
Total & 38 & 41 & 34 & 22 & 135 \\
\hline \multicolumn{1}{c}{ VDS } & No Pain & Mild Pain & Moderate Pain & Severe Pain & Total \\
\hline No Pain & $40(97.6 \%)$ & 1 & 0 & 0 & 41 \\
Mild Pain & 2 & $28(71.8 \%)$ & 8 & 1 & 39 \\
Moderate Pain & 0 & 9 & $19(59.4 \%)$ & 4 & 32 \\
Severe Pain & 0 & 2 & 5 & $16(69.6 \%)$ & 23 \\
Total & 42 & 40 & 32 & 21 & 135 \\
${ }^{*} p<0.001$ & & & & & \\
\hline
\end{tabular}

the original categorization scheme and 76.3 percent for the Bieri FPS, which indicates improvement over the original categorization scheme. Therefore, the research team decided to leave the NRS categorization scheme as originally proposed and to change the Bieri FPS categorization scheme to that suggested by the regression, basically tightening the moderate pain category on the Bieri FPS to face 4 only and expanding the severe pain category. These final equivalence schemes were tested on the validation sample.

Table 5 illustrates the correspondence between categorized VDS scores and the predicted NRS and Bieri FPS scores in the validation sample, the latter categorized according to the equivalence scheme from the regression analysis. Overall average percent agreement with the VDS in Table 5 was 92.6 percent for the NRS and 100 percent for the Bieri FPS. For comparison, overall average percent agreement in the validation sample between the categorized VDS and the other two scales with the original categorization scheme was 83.7 percent for the NRS and 71.9 percent for the Bieri FPS, which is higher than the agreement computed for the equivalence sample (70.4\% and 68.9\%, Table 3). Likewise for comparison, the overall average percent agreement in the validation sample between the categorized VDS and the 
Table 5.

Agreement $^{*}$ between Verbal Descriptor Scale (VDS) and predicted categorized Numeric Rating Scale (NRS) and Bieri Faces Pain Scale (PFS) (validation sample).

\begin{tabular}{|c|c|c|c|c|c|}
\hline \multirow{2}{*}{ VDS } & \multicolumn{5}{|c|}{ Predicted Categorized NRS } \\
\hline & No Pain & Mild Pain & Moderate Pain & Severe Pain & Total \\
\hline No Pain & 45 (100\%) & 0 & 0 & 0 & 45 \\
\hline Mild Pain & 0 & $26(72.2 \%)$ & 10 & 0 & 36 \\
\hline Moderate Pain & 0 & 0 & 26 (100\%) & 0 & 26 \\
\hline Severe Pain & 0 & 0 & 0 & 28 (100\%) & 28 \\
\hline Total & 45 & 26 & 36 & 28 & 135 \\
\hline \multirow{2}{*}{ VDS } & \multicolumn{5}{|c|}{ Predicted Categorized FPS } \\
\hline & No Pain & Mild Pain & Moderate Pain & Severe Pain & Total \\
\hline$\overline{\text { No Pain }}$ & $45(100 \%)$ & 0 & 0 & 0 & 45 \\
\hline Mild Pain & 0 & 36 (100\%) & 0 & 0 & 36 \\
\hline Moderate Pain & 0 & 0 & 26 (100\%) & 0 & 26 \\
\hline Severe Pain & 0 & 0 & 0 & 28 (100\%) & 28 \\
\hline Total & 45 & 36 & 26 & 28 & 135 \\
\hline
\end{tabular}

Bieri FPS with the equivalence categorization scheme was 76.3 percent for the Bieri FPS, which is identical to the value obtained for the equivalence sample (Table 4).

\section{DISCUSSION}

The Figure summarizes the correspondence we derived across the three pain scales. Although the VDS and Bieri FPS are depicted as linear and the NRS is not, we doubt that any of the three are strictly linear. The Figure illustrates that the clinical definitions of the pain categories are not at all linear. The no pain and moderate pain categories appear to be precisely defined in the VDS and Bieri FPS response scales. For the VDS, this outcome is likely an artifact of the word moderate in the response scale. The mild pain and severe pain categories are broader; more variation is noted in the original scale numbers included, which probably reflects the difficulty that residents have in quantifying pain with any tool.

We have demonstrated that a clinically relevant, usable categorization of pain that equates three different pain scales yields valid and reproducible assessments in the nursing home setting. A 10-year review of quality improvement monitoring in pain management suggested that standardized outcome measures are critically important [30]. Several reasons have been cited as to why determining a standardized metric for pain intensity and standardized cutoff points for pain-intensity levels across populations, disease conditions, and instruments is impor- tant. First, from a clinical perspective, having standardized pain-intensity measures (mild, moderate, severe) across different tools would help improve clinical decision-making regarding appropriate therapies for pain management; it would also facilitate communication about pain among clinicians and between clinician and patient [25]. Second, from a research perspective, a standardized metric for mild, moderate, and severe pain would provide greater confidence to investigators when comparing outcomes across groups for which different painintensity tools have been used [25]. Third, both clinicians and researchers would benefit if demonstrating that categorizations of mild, moderate, and severe pain corresponded reliably and accurately to three distinct ranges of pain severity as measured with the more refined quantitative instruments were possible [20]. Serlin et al. concluded from their studies that the nonlinear relationship that exists between reported pain severity and interference with activity is better captured by the identification of accurate cutoff points between mild, moderate, and severe pain than by the use of discrete numbers on the NRS [20].

From a policy perspective, the pain-intensity levels experienced by residents reflects nursing home quality and is used to target the nursing home for quality improvement efforts [31]. The Centers for Medicare and Medicaid Services define the pain quality indicator as the prevalence of residents with moderate pain every day or excruciating pain every day or less often. Wu et al. [32] and Teno et al. [33] labeled such residents as being in "severe pain." Nursing home staff is not told which pain-intensity tool to use for assessing residents' pain. In our study of nursing 


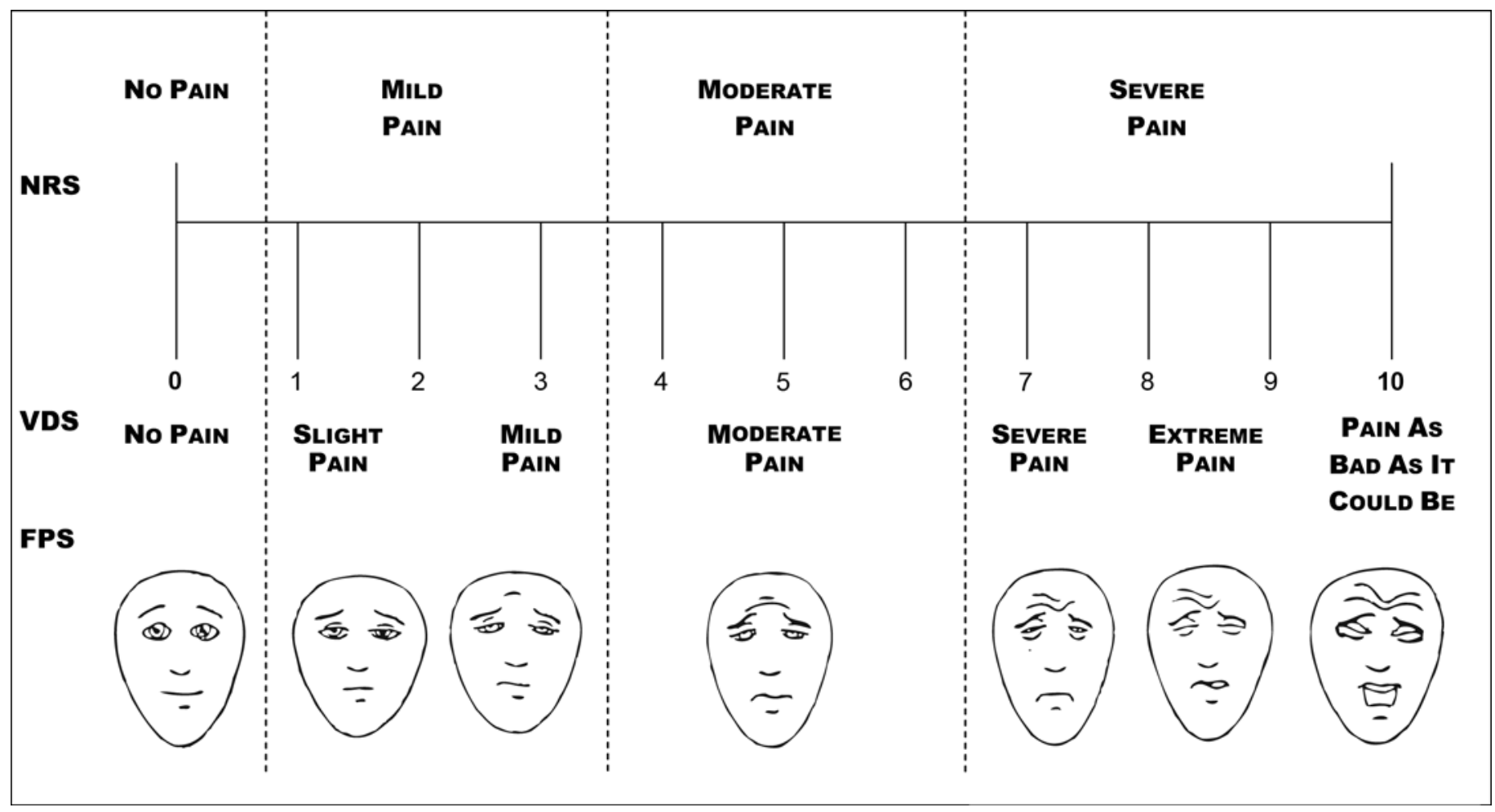

Figure.

Numeric Rating Scale (NRS), Verbal Descriptor Scale (VDS), and Faces Pain Scale (FPS). Source (FPS): Bieri D, Reeve RA, Champion GD, Addicoat L, Ziegler JB. The Faces Pain Scale for the self-assessment of the severity of pain experienced by children: Development, initial validation, and preliminary investigation for ratio scale properties. Pain. 1990;41(2):139-50. [PMID: 2367140] Used with permission.

homes, we found multiple tools in use, including $0-10$ and $0-5$ NRSs and several versions of the VDS and FPS. Wu et al. found that raters of pain in nursing homes were more likely to agree on the presence of pain than the level of pain; the authors attributed this to rater experience and/or expectation, as well as the use of imprecise intensity scales [32]. We do not know how individual raters translate the results of the pain-intensity assessments from these diverse tools to the broad categorizations of mild, moderate, and severe pain or whether different raters use the same metric in these conversions within or across nursing homes.

Perhaps even more important, individuals within each group (mild, moderate, or severe pain) require different types of pain treatment [10]. Faulty interpretation of the meaning and impact of a pain-rating categorization on a standardized pain-intensity scale may lead to inappropriate treatment [34]. A meaningful threefold grading of pain severity would allow definition of a quantifiable target range of adequate pain relief [20]. In other words, we would know which number, face, or words we were aiming for in terms of pain relief. The severity groupings that were derived in this study could be incorporated into the APS guidelines [10] to better guide selection of appropriate analgesia.

Our analysis revealed that extensive variability exists in individual responses to pain, undoubtedly because of individual interpretations of the faces, numbers, and verbal descriptors as indicators of pain. This variability is at least partly responsible for the agreement difference between the randomly selected equivalence and validation samples. None of the scales is a perfect measure of an inherently subjective experience. An 11-point NRS may be too detailed for practical clinical use in the nursing home setting. In addition, given the evidence that residents choosing "moderate" pain on the VDS tended to choose "mild" pain on the Bieri FPS and the reverse for "mild pain" on the VDS, the faces on the Bieri FPS may be too difficult for most residents to use as a painintensity measure, although those with language difficulties appeared to prefer it and should be given it as an option. 


\section{CONCLUSIONS}

Accurate assessment of pain intensity in nursing home residents can be accomplished using several different standardized pain-intensity tools. Nursing home residents generally prefer the VDS, but select groups have been shown to prefer the NRS or FPS. Available clinical practice guidelines present a variety of possible pain-intensity tools for use with older adults and in nursing homes, and individual nursing homes have adopted a wide range of tools for their use. The equated pain intensity scores derived from these multiple tools can be used to guide treatment decisions, evaluate effectiveness of the selected therapy, and reflect the quality of pain management within the facility. Regardless of which pain-intensity tool is used, the categorization of mild, moderate, and severe pain derived from this analysis provides the best evidence thus far for the correspondence of pain-intensity levels among three wellknown pain-intensity scales used with older adults: Bieri FPS, VDS, and NRS.

This study was limited to nursing home residents in one state, and the current analysis was limited to residents who were able to verbalize their pain. However, generalizability is enhanced by the fact that equal numbers of rural and urban homes were involved in the project and that a meaningful number of Hispanic subjects were represented. Whether the equivalence shown here is applicable to noninstitutionalized older adults and younger patients in pain should be evaluated.

\section{ACKNOWLEDGMENTS}

Dr. Jones is now with the Frances Payne Bolton School of Nursing, Case Western Reserve University, Cleveland, Ohio.

This material was based on work supported by the Agency for Healthcare Research and Quality (grant U18HS11093 to the School of Nursing, University of Colorado at Denver and the Health Sciences Center, Denver, Colorado; principal investigator Katherine R. Jones).

The authors have declared that no competing interests exist.

\section{REFERENCES}

1. Cohen-Mansfield J, Creedon M. Nursing staff members' perceptions of pain indicators in persons with severe dementia. Clin J Pain. 2002;18(1):64-73. [PMID: 11803305]
2. Weiner D, Herr K. Comprehensive interdisciplinary assessment and treatment planning: An integrative overview. In: Weiner D, Herr K, Rudy TE, editors. Persistent pain in older adults: An interdisciplinary guide for treatment. New York (NY): Springer; 2002. p. 18-57.

3. Ferrell BA. Overview of aging and pain. In: Ferrell BR, Ferrell BA, editors. Pain in the elderly: A report of the task force on pain in the elderly of the International Association for the Study of Pain. Seattle (WA): IASP Press; 1996. p. 1-10.

4. Sengstaken EA, King SA. The problems of pain and its detection among geriatric nursing home residents. J Am Geriatr Soc. 1993;41(5):541-44. [PMID: 8486889]

5. Won AB, Lapane KL, Vallow S, Schein J, Morris JN, Lipsitz LA. Persistent nonmalignant pain and analgesic prescribing patterns in elderly nursing home residents. J Am Geriatr Soc. 2004;52(6):867-74. [PMID: 15161448]

6. Ferrell BA, Ferrell BR, Osterweil D. Pain in the nursing home. J Am Geriatr Soc. 1990;38(4):409-14. [PMID: 2109765]

7. Chodosh J, Solomon DH, Roth CP, Chang JT, MacLean $\mathrm{CH}$, Ferrell BA, Shekelle PG, Wenger NS. The quality of medical care provided to vulnerable older patients with chronic pain. J Am Geriatr Soc. 2004;52(5):756-61. [PMID: 15086657]

8. American Geriatrics Society (AGS).The management of persistent pain in older persons: AGS Panel on Persistent Pain in Older Persons. J Am Geriatr Soc. 2002;50(6 Suppl): S205-24. [PMID: 12067390]

9. Closs SJ, Barr B, Briggs M, Cash K, Seers K. A comparison of five pain assessment scales for nursing home residents with varying degrees of cognitive impairment. J Pain Symptom Manage. 2004;27(3):196-205. [PMID: 15010098]

10. Miaskowski C, Cleary J, Burney R, Coyne P, Finley R, Foster R, Grossman S, Janjan N, Ray J, Syejala K, Weisman S, Zahrbock C. Guideline for the management of cancer pain in adults and children. 3rd ed. Glenview (IL): American Pain Society; 2005. p. 1-166.

11. Herr K. Pain assessment in cognitively impaired older adults. Am J Nurs. 2002;102(1):65-67. [PMID: 12473932]

12. Herr K, Bjoro K, Decker S. Tools for assessment of pain in nonverbal older adults with dementia: A state-of-the-science review. J Pain Symptom Manage. 2006;31(2):170-92. [PMID: 16488350]

13. Herr K, Coyne PJ, Key T, Manworren R, McCaffery M, Merkel S, Pelosi-Kelly J, Wild L, American Society for Pain Management Nursing. Pain assessment in the nonverbal patient: Position statement with clinical practice recommendations. Pain Manag Nurs. 2006;7(2):44-52. [PMID: 16730317]

14. Herr KA, Mobily PR. Comparison of selected pain assessment tools for use with the elderly. Appl Nurs Res. 1993; 6(1):39-46. [PMID: 8439177]

15. Dalton JA, McNaull F. A call for standardizing the clinical rating of pain intensity using a 0 to 10 rating scale. Cancer Nurs. 1998;21(1):46-49. [PMID: 9494230] 
16. Jones KR, Fink R, Hutt E, Vojir C, Pepper GA, Scott-Cawiezell J, Mellis BK. Measuring pain intensity in nursing home residents. J Pain Symptom Manage. 2005;30(6):519-27. [PMID: 16376738]

17. American Geriatrics Society (AGS). The management of chronic pain in older persons: AGS Panel on Chronic Pain in Older Persons. J Am Geriatr Soc. 1998;46:635-51. [PMID: 9588381] Erratum in: J Am Geriatr Soc. 1998;46(7):913.

18. American Medical Directors Association. Chronic pain management in the long-term care setting: Clinical practice guideline. Baltimore (MD): American Medical Directors Association; 1999.

19. Paul SM, Zelman DC, Smith M, Miaskowski C. Categorizing the severity of cancer pain: Further exploration of the establishment of cutpoints. Pain. 2005;113(1-2):37-44. [PMID: 15621362]

20. Serlin RC, Mendoza TR, Nakamura Y, Edwards KR, Cleeland CS. When is cancer pain mild, moderate or severe? Grading pain severity by its interference with function. Pain. 1995;61(2):277-84. [PMID: 7659438]

21. Jensen MP, Smith DG, Ehde DM, Robinson LR. Pain site and the effects of amputation pain: Further clarification of the meaning of mild, moderate, and severe pain. Pain. 2001;91(3):317-22. [PMID: 11275389]

22. Turner JA, Franklin G, Heagerty PJ, Wu R, Egan K, Fulton-Kehoe D, Gluck JV, Wickizer TM. The association between pain and disability. Pain. 2004;112(3):307-14. [PMID: 15561386]

23. Zelman DC, Hoffman DL, Seifeldin R, Dukes EM. Development of a metric for a day of manageable pain control: Derivation of pain severity cut-points for low back pain and osteoarthritis. Pain. 2003;106(1-2):35-42. [PMID: 14581108]

24. Mendoza TR, Chen C, Brugger A, Hubbard R, Snabes M, Palmer SN, Zhang Q, Cleeland CS. Lessons learned from a multiple-dose post-operative analgesic trial. Pain. 2004; 109(1-2):103-9. [PMID: 15082131]

25. Palos GR, Mendoza TR, Mobley GM, Cantor SB, Cleeland CS. Asking the community about cutpoints used to describe mild, moderate, and severe pain. J Pain. 2006; 7(1):49-56. [PMID: 16414555]
26. Krulewitch H, London MR, Skakel VJ, Lundstedt GJ, Thomason $\mathrm{H}$, Brummel-Smith $\mathrm{K}$. Assessment of pain in cognitively impaired older adults: A comparison of pain assessment tools and their use by nonprofessional caregivers. J Am Geriatr Soc. 2000;48(12):1607-11. [PMID: 11129750]

27. Bieri D, Reeve RA, Champion GD, Addicoat L, Ziegler JB. The Faces Pain Scale for the self-assessment of the severity of pain experienced by children: Development, initial validation, and preliminary investigation for ratio scale properties. Pain. 1990;41(2):139-50. [PMID: 2367140$]$

28. Jones KR, Fink R, Vojir C, Pepper G, Hutt E, Clark L, Scott J, Martinez R, Vincent D, Mellis BK. Translation research in long-term care: Improving pain management in nursing homes. Worldviews Evid Based Nurs. 2004;1 Suppl:S13-20. [PMID: 17129330]

29. Jones KR, Fink R, Pepper G, Hutt E, Vojir CP, Scott J, Clark L, Mellis K. Improving nursing home staff knowledge and attitudes about pain. Gerontologist. 2004;44(4): 469-78. [PMID: 15331804]

30. Gordon DB, Pellino TA, Miaskowski C, McNeill JA, Paice JA, Laferriere D, Bookbinder M. A 10-year review of quality improvement monitoring in pain management: Recommendations for standardized outcome measures. Pain Manag Nurs. 2002;3(4):116-30. [PMID: 12454804]

31. Teno JM, Kabumoto G, Wetle T, Roy J, Mor V. Daily pain that was excruciating at some time in the previous week: Prevalence, characteristics, and outcomes in nursing home residents. J Am Geriatr Soc. 2004;52(5):762-67. [PMID: 15086658]

32. Wu N, Miller SC, Lapane K, Gozalo P. The problem of assessment bias when measuring the hospice effect on nursing home residents' pain. J Pain Symptom Manage. 2003;26(5):998-1009. [PMID: 14585551]

33. Teno JM, Weitzen S, Wetle T, Mor V. Persistent pain in nursing home residents. JAMA. 2001;285(16):2081. [PMID: 11311096]

34. McNeill JA, Sherwood GD, Starck PL. The hidden error of mismanaged pain: A systems approach. J Pain Symptom Manage. 2004;28(1):47-58. [PMID: 15223084$]$

Submitted for publication May 26, 2006. Accepted in revised form October 25, 2006. 